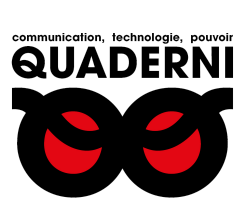

\title{
Quaderni
}

Communication, technologies, pouvoir

\section{Étude des processus de radicalisation au sein des réseaux sociaux : place des arguments complotistes et des discours de rupture}

Study of radicalization processes within social networks: role of complotist

arguments and rupture discourses

\section{Séraphin Alava, Noha Najjar et Hasna Hussein}

\section{(2) OpenEdition \\ Journals}

\section{Édition électronique}

URL : https://journals.openedition.org/quaderni/1106

DOI : 10.4000/quaderni.1106

ISSN : 2105-2956

\section{Éditeur}

Les éditions de la Maison des sciences de l'Homme

Édition imprimée

Date de publication : 5 octobre 2017

Pagination : 29-40

\section{Référence électronique}

Séraphin Alava, Noha Najjar et Hasna Hussein, «Étude des processus de radicalisation au sein des réseaux sociaux : place des arguments complotistes et des discours de rupture », Quaderni [En ligne], 94 | Automne 2017, mis en ligne le 05 octobre 2019, consulté le 22 juillet 2021. URL : http:// journals.openedition.org/quaderni/1106; DOI : https://doi.org/10.4000/quaderni.1106 


\section{$D$ ossier}

Étude des processus de radicalisation

\section{au sein des réseaux} sociaux : place des arguments complotistes et des discours de rupture

Séraphin

Alava

Professeur en sciences de l'éducation, Université de Toulouse 2 - Jean Jaurès EFTS

Noha

Najjar

Docteure en sciences de l'éducation Université de Toulouse 2 - Jean Jaurès Chercheuse associée EFTS

\section{Hasna Hussein}

Docteure en sociologie des médias Université de Toulouse 2 - Jean Jaurès
Dans cette stratégie discursive et d'endoctrinement, les théories du complot agissent en synergie avec d'autres thèmes issus des discours de rupture qui favorisent le questionnement du sujet et l'entrée en quête d'un sens caché. À travers un corpus d'interaction langagière issu volontairement des interactions sur Facebook (12 personnes en voie de radicalisation et 6 personnes sorties de ce processus) et l'étude lexicométrique des discours publics des groupes radicaux (jihadistes, racistes), nous montrerons comment le champ sémantique, les thèmes discursifs, les stratégies de communication fonctionnent dans la déconstruction du réel, dans l'isolement de la pensée et dans la réduction de la complexité. Notre travail vise à montrer que la communication en réseaux accélère les processus cognitifs en jeu dans cette phase essentielle de la conviction radicale. Nous souhaitons décrire et expliquer le processus paradoxal d'éducation sombre qui est actif au sein des médias sociaux aujourd'hui.

Non coupable mais responsable : que dire des liens entre réseaux sociaux et radicalisation.

Chaque jour et notamment après des attentats terroristes se repose la question de la responsabilité du Cyberespace dans la radicalisation. Est-ce que les réseaux sociaux conduisent les individus vulnérables à recourir à la violence ? Est-ce qu'un processus d'auto radicalisation est en cours sur le Net et comment s'en prévenir ? Partout dans le monde, les gouvernements et les entreprises d'Internet prennent des décisions sur la base de cette hypothèse sur un lien causal entre présence sur le Web ou au sein des réseaux de contenus radicaux, voire d'apologie du terrorisme et sur le développement des attaques violentes. L'enjeu 
est de taille tant le cyberespace est devenu un espace essentiel et central de communication dans le monde. L'Internet est-il coupable de cette montée des discours radicaux, de l'émergence de la violence brutale et radicale? Il ne sert à rien de nier l'évidence et il est facile de constater que :

a. Les discours violents discriminatoires, racistes se diffusent facilement sur le Net malgré les censures et les interdictions ;

b. Des groupes volontaires agissent de plus en plus pour relayer ces messages et la « complotsphère » est très active dans les médias classiques et sur les réseaux sociaux ;

c. Des recruteurs formés et efficaces sont présents dans les réseaux publics ou cryptés pour accrocher les jeunes et les enrôler dans leurs univers radicaux et violents.

La lutte contre ces trois dimensions (médiatique, communicationnelle et intersubjective) est exigeante. C'est dans ce contexte que l'UNESCO a commandé à notre laboratoire une étude intitulée Social Media and Radicalization of Youth in the Digital Age. Ce travail conduit en 2016 et 2017 fournit une cartographie globale de la recherche (principalement entre 2012 et 206) dans les rôles assumés par les médias sociaux dans les processus de radicalisation violente, en particulier lorsqu'ils affectent les jeunes et les femmes. Cette recherche confirme la conviction que l'Internet dans son ensemble est souvent considéré comme un vecteur actif pour la radicalisation violente qui facilite la prolifération des idéologies extrémistes violentes. Les recherches internationales analysées montrent que les groupes extrémistes sont en effet très répandus sur Internet et qu'il existe une véritable stratégie de séduction et de persuasion radicale qui s'est facilement adaptée aux spécificités d'un monde numérique global. Il existe de plus en plus de connaissances sur la façon dont les terroristes utilisent le cyberespace. L'impact de cette stratégie est toutefois moins clair sur les individus et surtout il est impossible d'établir un lien de causalité entre la lecture de ces messages et les passages à l'acte violent ou les radicalisations observables. À la suite de ce travail nous avons voulu aller plus loin et comprendre les raisons d'une efficacité des messages, médias et conversations radicales sur les « jeunes». Nous avons aussi voulu préciser le rôle des arguments dans le processus communicationnel du recruteur et de l'embrigadé et notamment la place des discours complotistes et de rupture.

En effet en ce qui concerne les théories de l'impact des médias, nous refusons à la fois la théorie de la « chambre d'écho » qui renvoie l'origine de la radicalisation à l'extérieur et qui donne aux réseaux sociaux un rôle d'amplificateurs d'une réalité existant dans la vie réelle, et à la fois la théorie dite de la « seringue hypodermique » qui évoque un effet massif des réseaux qui anesthésie les consciences et rend les usagers totalement dociles aux influences radicales (Ducol, 2015, Lohisse 2006). Nos recherches mettent en avant une relation plus systémique et communicationnelle entre les réseaux sociaux et les sujets radicalisés. En effet les médias sociaux ont des spécificités à prendre en compte qui permettent de produire des process communicationnels qui vont instruire une scène communicationnelle favorable à la persuasion / engagement ou à la démarche de manipulation / persuasion. 
Rappelons que le processus de radicalisation est un processus conversationnel qui, partant des préoccupations des jeunes, va avancer vers des idées radicales et de rupture. Au cœur de ces approches, il y a une interprétation d'événements, de prises de position politiques ou sociales qui vont être présentés sous l'angle fermé de l'endoctrinement. Les jeunes n'ayant pas une véritable éducation à l'information et aux médias vont facilement se laisser convaincre. Les digital natives sont, bien souvent, des digital naïs incapables de distinguer les informations objectives issues de sites officiels, d'articles scientifiques et les informations issues de sites conspirationnistes ou suprématistes. L'absence d'une véritable éducation aux médias est un élément facilitant l'embrigadement. Le jeune fonctionne souvent en « clôture informationnelle » (Bougnoux, 1995) qui le pousse à privilégier d'abord les informations qui sont conformes à son idée première.

\section{L'Internet est devenu peu à peu le domaine privilégié des discours de haine. Dans les} posts, dans les forums, les discours xénophobes, homophobes, antisémites ou islamophobes se déploient de plus en plus. Les jeunes voient donc s'amplifier une littérature qui banalise la haine et la violence. Toutes les études sur la cyber violence ou la cyber haine nous alertent sur ce point (Gendarmerie royale du Canada, 2009) et les jeunes sont très sensibles à ces arguments, car la violence entre eux est banalisée. L'absence d'une politique forte de contre-discours ou de narrations citoyennes laisse donc la place aux théories complotistes et aux théories suprématistes.

La clôture informationnelle renforce alors la création d'une « bulle cognitive " qui se met de façon cyclique à renforcer la crédibilité de l'information qui justifie la pensée clôture. $\mathrm{Si}$ nous ajoutons à cela l'idée de "l'entre soi » privilégié par les jeunes et les adultes dans leurs communications numériques, alors la clôture est aussi sociale et le recruteur réussit alors le triple enfermement (une méfiance devant l'information, une fermeture de la pensée, une structuration d'un groupe commun d'adhésion et de communication).

En troisième lieu, la radicalisation n'est pas un processus d'endoctrinement, mais de conversion et d'adhésion. En effet, il ne faut pas la penser comme un processus passif pour le jeune où des recruteurs hypnotisent des jeunes victimes impuissantes. Non, la radicalisation numérique est une démarche à deux d'accroche, de demandes, de convictions et de doutes. Comme nous l'avons indiqué, la démarche de radicalisation ne commence pas par la présence sur l'Internet de sites terroristes ou des médias développant des discours raciaux ou terroristes. Elle commence par des conversations s'appuyant sur de réelles préoccupations des jeunes qui exposent leurs opinions, leurs questionnements au sein des réseaux sociaux. L'《extimité » des jeunes, c'est-à-dire leur propension à exprimer à des personnes inconnues les faits les plus intimes est une source de cyber violence. Cette extimité est utilisée par les recruteurs pour accrocher leurs proies et construire un lien affectif très fort (parfois amoureux), entrainant le jeune dans une dépendance affective qui favorisera son adhésion aux idéaux et aux mythes nocifs proposés par le recruteur.

Enfin, le quatrième point qui facilite le bascu- 
lement est le caractère particulièrement persuasif de la communication numérique où les éléments fondateurs de la communication au sens de Watzlawick sont en interaction permanente. Il existe donc une véritable séduction numérique qui entre en écho intime avec l'individu et qui va ouvrir sa conscience à des idées qui sont aujourd'hui présentes dans le débat public de la jeunesse (théories complotistes, discours de rupture, discours religieux, discours moral).

\section{La conversation d'engagement radical : commu- niquer, c'est conduire et manipuler}

Pour comprendre cela, il est nécessaire de se référer aux approches des théories de Palo Alto qui examine la notion de communication persuasive et qui, partant d'axiomes structurants, développe l'idée selon laquelle « il n'y a pas de relation humaine qui ne soit soumise à l'influence [...] il n'y a pas [...] de relation sans manipulation », Watzlawick (1980). Cette affirmation peut paraître très osée tant nous sommes encore convaincus du caractère objectif de la communication (Jakobson) où le contenu du message est indépendant du média (Macluhan) et indépendant des sujets qui le reçoivent. En fait l'école de Palo Alto et Watzlawick ont montré combien le message est un construit social dépendant grandement du couple émetteur-récepteur et surtout que la relation (communication) est aussi un élément constitutif du contenu (information) (Bougnoux 1995). En ce sens, communiquer c'est participer à la production du sens en construisant dans l'interaction un processus de communication. C'est donc au sens instrumental du terme une manipulation c'est-à-dire une opération de conduite. Cette analyse pose donc le principe de la conduite comme une opération indispensable à la fois au récepteur et à l'émetteur. En effet si dans une conversation Facebook deux sujets dialoguent, ils agissent de façon intersubjective en manipulant la situation. La communication est donc par essence «persuasive » quand elle recherche à la fois de l'« efficacité » et de l'« efficience », c'est-à-dire à la fois des effets voulus sur autrui par l'auteur d'un message et d'autres que celui-ci n'a pas « consciemment » recherchés.

\section{La conversation radicale, une pragmatique de la communication en réseau}

Cette particularité de la communication comme processus intersubjectif permet alors à la fois de comprendre la spécificité des communications en réseau mais aussi l'échec des politiques médiatiques dites de contre-discours. En effet, comme le démontre Philippe Breton (2008), le transfert efficace d'une information par le seul jeu du raisonnement est un leurre et les campagnes de contre-discours s'appuient fortement sur l'idée que le message djihadiste peut être dénoncé et surtout invalidé par l'argumentation. La rhétorique comprise comme la science de la communication persuasive prend alors en compte d'autres éléments déterminant l'engagement du sujet dans l'acte de communication et valide une pragmatique de la communication comme scène médiatique subjective et humaine.

Dans l'étude des scripts d'interactions que nous avons examinés, dans l'ensemble des récits de radicalisation que nous avons analysés, le moment du dialogue est à la fois un moment communicationnel, émotionnel, intersubjectif qui fonde un ici et maintenant où le sujet se 
perçoit comme acteur même s'il agit en dehors de sa conscience. L'analyse des spécificités des conversations sur les réseaux sociaux renforce l'idée que la « relation englobe le contenu » (Watzlawick, Helmick Beavin, Don D. Jackson, 1972). Si toute communication englobe ces deux aspects, la communication virtuelle potentialise ces deux éléments à travers les notions d'identités virtuelles, d'extimité et performativité des mots pris dans un univers de relation. Si je construis dans l'interaction le contenu des messages, je fais cela aussi en référence à une personnalité virtuelle vers laquelle je construis aussi des significations et dans un feed back permanent de ce que je confie à l'autre. Cette circularité communicationnelle où l'auteur d'un message est construit par le sens que je donne au message et par la confiance que je lui attribue de par mon intimité partagée est une dynamique essentielle de la « séduction numérique » qui est en jeu dans les scripts que nous avons analysés. La force des idées complotistes partagées au sein des conversations radicales réside dans trois principes que Watzlawick a parfaitement analysés :

a. Le contenu et la relation : l'idée complotiste, djihadiste que je reçois est faite de la relation virtuelle que je construis avec le sujet manipulateur. Cette relation est unique et essentielle car elle révèle des éléments cachés aux autres. Je suis donc un être unique et l'idée complotiste s'en trouve alors validée par la force de ma relation.

\section{b. La communication humaine utilise simul- tanément deux modes de communication, digital et analogique : le mode de transmission des idées radicales, djihadistes ou complotistes est}

un mode de transmission fortement analogique, c'est-à-dire qui s'appuie sur des exemples concrets et donne du sens à tous ces exemples parfois lointains et dénués de sens. Comme ce que tu dis s'illustre par ce cas, alors la théorie est valide. De plus, comme l'explication est ésotérique, je suis placé dans la situation de l'initié qui met enfin en lien des signes concrets épars. Le sens digital c'est-à-dire conceptuel est alors validé.

\section{c. La nature d'une relation dépend de la ponctuation des séquences de communication :} les discours djihadistes, complotistes et radicaux se transmettent au sein de conversations longues souvent dans des temps privés, parfois à l'insu des autres, toujours dans des temps où l'émotion est forte. Ces rencontres entre le recruteur et le recruté sont attendues et le rituel est accentué par l'impossibilité de connaître la source du message et aussi le caractère prohibé de la communication (il ne faut en parler à personne). La relation devient alors vite addictive et cette addiction renforce l'efficacité de la communication. Les contenus transmis sont alors validés par la caractéristique exceptionnelle de la communication.

\section{Approche psychosociale de l'embrigadement radical}

Bien que la radicalisation horizontale et autonome soit de plus en plus soulignée par plusieurs études (Heinkel \& Mace, 2011), le rôle du portier ou du recruteur reste très influent. Il constitue le pont entre la personne en voie de radicalisation et les groupes extrémistes, et fournit le « savoirfaire » afin de joindre une cellule, acquérir l'entrầnement nécessaire et la connaissance concernant la politique et la religion (Nesser, 2006). 
Cependant, le recruteur n'active pas le processus de radicalisation, il permet plutôt de l'accélérer (Verldhuis et Bakker, 2007).

À ce niveau-là, la personne qui sera recrutée subit une « triple manipulation » : comportementale, mentale et morale ou affective. Sur le plan comportemental, la personne recrutée sera amenée à accomplir de petits gestes, des actes anodins, puis de plus en plus accaparants, coercitifs afin qu'elle perde son libre-arbitre et commence à adopter en toute liberté des comportements nouveaux qui sont ceux du « formateur » ou « recruteur ». Sur le plan des idées de « manipulation cognitivementale », le matraquage, le bombardement intellectuel à partir de séances de prières, de stages, de séminaires, de lectures intensives, rendent la personne en voie de radicalisation plus réceptive à de nouvelles idées et visions du monde. Ce processus devient plus facile quand la personne visée est victime de crises de nature économique (par ex. perte d'emploi), socioculturelle (par ex. humiliation, racisme, etc.), politique (par ex. torture, discrimination politique, répression) ou personnelle (par ex. mort d'un membre de la famille). Certains membres du groupe vont profiter de ces crises existantes chez les personnes et vont les amplifier encore pour que celles-ci rejoignent leur cause. La troisième persuasion, affective et morale, introduit le futur radicalisé dans une nouvelle famille au détriment de la famille biologique. Cette nouvelle famille se montre extrêmement chaleureuse et procure un sentiment d'appartenance qui remplacera celui de la société et de la communauté. Une nouvelle identité se constitue, des idées, des comportements, des pratiques et des sentiments nouveaux prennent place.

\section{Méthodologie de recueil des récits, base de données des parcours, approche en sociologie clinique}

Dans la stratégie discursive et d'endoctrinement mise en œuvre par des recruteurs ou des militants radicaux sur le Net, les théories du complot agissent en synergie avec d'autres thèmes issus des discours de rupture qui favorisent le questionnement du sujet et l'entrée en quête d'un sens caché. L'opérateur de la manipulation construit pour chaque sujet un espace et une démarche de communication personnalisée qui s'adapte au sujet et à la situation. Pour analyser cela, nous avons choisi de travailler sur un corpus d'interaction langagière issu volontairement des interactions sur Facebook (12 personnes en voie de radicalisation et 6 personnes sorties de ce processus).

Nous avons ensuite replacé ces scripts de conversation dans la perspective des récits recueillis auprès de ces personnes lors d'entretiens s'appuyant sur les méthodes qualitatives de la sociologie clinique et des histoires de vies. Le récit de vie est comme mode d'investigation des conduites humaines et des processus sociaux bien adapté au phénomène de la radicalisation car cette méthode est à la fois un outil de recherche mais aussi un outil d'intervention sociale. Nous cherchons donc durant des entretiens longs non directifs à poser les étapes des processus et à faire émerger par le sujet les points d'étapes qui semblent marquer les phases de l'embrigadement. Dans tous les cas de radicalisation que nous avons eu à traiter, la dimension numérique est présente mais nous avons choisi 12 cas qui portent les trois dimensions de la radicalisation numérique caractéristique (contact direct de personnes sur le 
net sans contact préalable dans la vie réelle, envoi de liens documentaires et recherche volontaire d'information par le sujet, groupe d'adhésion numérique élargie).

Ensuite et avec l'accord des personnes, nous avons eu accès aux scripts des interactions, aux sites consultés et au récit reconstruit des interactions. Ce sont ces éléments bruts que nous avons exploités en analysant le champ sémantique, les thèmes discursifs, les stratégies de communication utilisés par l'émetteur et le récepteur et nous avons ensuite constitué des graphes d'interaction et de sens pour analyser comment le recruteur agissait pour assurer un isolement de la personne, une déconstruction du réel, la submersion affective de la raison et la réduction de la complexité.

Dans les 12 cas analysés, 8 sont des cas de jeunes filles et 4 des cas de garçons. Nous avons recueilli des éléments quantitatifs pour analyser leurs profils (le nom et l'origine des personnes sont modifiés)
Les profils d'usages des jeunes sont calculés à partir de l'échelle des usages numériques de Bernoff qui permet de caractériser les usages numériques dominants. Dans cette échelle, nous distinguerons le profil de « communicant actif» marqué par une présence importante sur les réseaux (plus de 4 réseaux sociaux et plus de $70 \%$ du temps numérique passé en communication). Le profil « joueur actif » est caractérisé par une centration des pratiques numériques autour des jeux en ligne et autour de relations en réseaux autour du jeu. Le profil collecteur actif est caractérisé par une présence sur le Net active dans des blogs, les commentaires des médias et sur les sites d'expression citoyenne (écologie, antiracisme, égalité des droits, etc.).

Nous constatons à partir de ces analyses trois éléments importants : les parcours de radicalisation numérique ne sont en corrélation ni avec une orientation religieuse, ni avec une pratique numérique spécifique, ni avec la présence préalable d'idées complotistes. Par contre, l'existence

\begin{tabular}{|c|c|c|c|c|c|}
\hline Prénom & Genre & Famille & Idées complotistes & Usages numériques & Age \\
\hline Asma & Féminin & Famille musulmane & Non présentes & Communicante active & 13 \\
\hline Sonia & Féminin & Famille musulmane & Non présentes & Joueuse active & 16 \\
\hline Pierre & Masculin & Famille athée & Non présentes & Communicante active & 18 \\
\hline Aminata & Féminin & Famille musulmane & Déjà présentes & Communicante active & 15 \\
\hline Julia & Féminin & Famille athée & Non présentes & Collectrice engagée & 15 \\
\hline Lucas & Masculin & Famille catholique & Déjà présentes & Joueur actif & 20 \\
\hline Nina & Féminin & Famille athée & Déjà présentes & Communicante active & 14 \\
\hline Clément & Masculin & Famille catholique & Non présentes & Joueur actif & 15 \\
\hline Aziz & Masculin & Famille musulmane & Déjà présentes & Joueur actif & 16 \\
\hline Yona & Féminin & Famille catholique & Non présentes & Communicante active & 14 \\
\hline Louna & Féminin & Famille athée & Non présentes & Communicante active & 14 \\
\hline Thaïs & Féminin & Famille athée & Non présentes & Collectrice engagée & 17 \\
\hline
\end{tabular}

Tableau 1 : caractéristiques sociales des sujets 
d'une pratique numérique active (plus de $9 \mathrm{~h}$ par semaine) est corrélée avec un parcours de radicalisation.

L'étude des scripts de radicalisation numérique montre l'existence d'une véritable stratégie de manipulation que nous illustrons par cette cible.

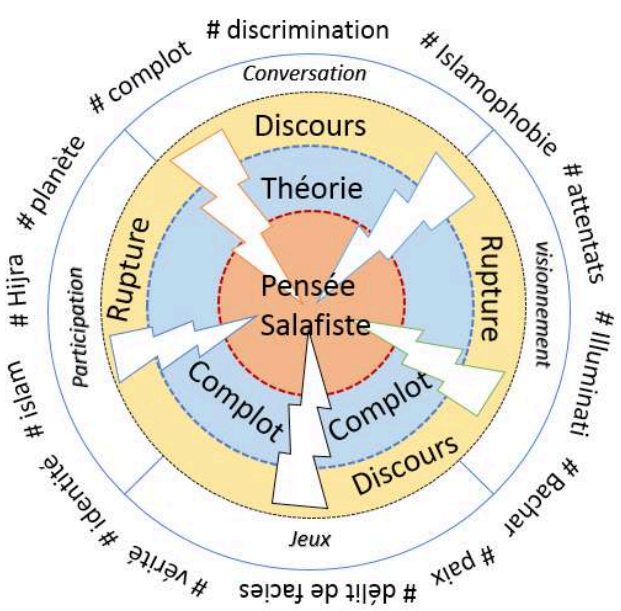

Figure1: Cible de l'embrigadement numérique djihadiste

En effet s'il n'existe pas de facteurs cohérents pour expliquer la radicalisation numérique des jeunes, il existe une démarche en quatre étapes qui se retrouve toujours dans l'analyse des scripts.

Dans la phase 1, la cible est identifiée par une expression caractérisant ses opinions, interrogations, prises de position sur le Net. C'est soit sur twitter dans une indexation d'un fait, soit dans un commentaire sur un site d'information ou d'alter information, soit dans le post ou le commentaire fait sur Facebook. Ces mots-clés d'entrée ne sont pas exhaustifs mais ils marquent tous une ouverture possible à des informations centrées sur des discours de rupture.

La phase 2 est alors possible, d'abord par «l'accrochage de l'attention» fait par le recruteur par un post, un j'aime, un soutien explicite dans un retweet, etc. L'objectif est bien d'hameçonner le sujet en montrant une adhésion à ses idées. D'abord "le message est un massage », dirait McLuhan. Dans cette phase, la quête essentielle du recruteur est de construire les éléments structurants de la scène médiatique qui doit servir ensuite. Il s'agit alors de créer une complicité, un intérêt réciproque, une attirance construite sur la valorisation du sujet-cible. La scène médiatique comprend aussi une construction rituélique du contact, un espace spécifique privé du dialogue qui pose le ici et maintenant et le toi, et rien que toi, comme structurant la communication.

La conversation radicale va donc alors commencer et elle se structure dans la plupart des cas en trois phases (discours de rupture - théorie du complot - discours salafiste). Dans le cas des non-musulmans, la conversion viendra ancrer le sujet dans une acceptation de la perte de soi en faveur du collectif.

a. Les discours de rupture montrent au jeune que ses intérêts, ses indignations, ses questions sont des éléments communs à tous ceux qui comme lui sont ségrégés.

b. La théorie du complot est toujours présente dans le script de la conversation radicale. L'analyse des conversations numériques nous a permis d'identifier 5 formes spécifiques d'utilisation de ces théories du complot. 
1) Fonction invalidante : la théorie du complot vient dire « tout est faux » « tout est apparence » et donc la théorie du complot invalide les formes du raisonnement préalable pour ancrer la relation cognitive dans une dénonciation du visible et dans une quête du vrai invisible.

2) Fonction déictique : le complot joue le rôle de révélateur. Il montre que ce qui est vu n'est pas le vrai et donc qu'il y a un plan caché ésotérique à percevoir. Le complot ouvre un univers du sens caché et donc détourne des sens apparents et rationnels.

3) Fonction émotive : il s'agit par la révélation d'un complot ou par la confirmation d'un complot, de rapprocher l'émetteur du destinataire. L'émetteur révèle le complot car il est proche de l'émetteur. Il rompt le secret pour alerter, prévenir et donc rassurer le destinataire et lui montrer qu'il est pour lui comme un frère ou une sœur. À la sortie ils partagent ensemble une connaissance ésotérique. Ils sont de la même communauté de dénonciation.

4) Fonction d'assignation : la théorie du complot et sa révélation ou sa confirmation assignent au récepteur le rôle de victime et permettent au recruteur de montrer que, tout en étant manipulés, nous devons retrouver notre capacité à décider par l'action.

5) Fonction mobilisatrice : grâce à la théorie du complot et à sa révélation, il existe donc une urgence pour soi mais surtout pour les pairs qui sont eux aussi trompés d'agir pour rompre ce cercle de résignation et de victimisation. L'action de rupture est alors essentielle.

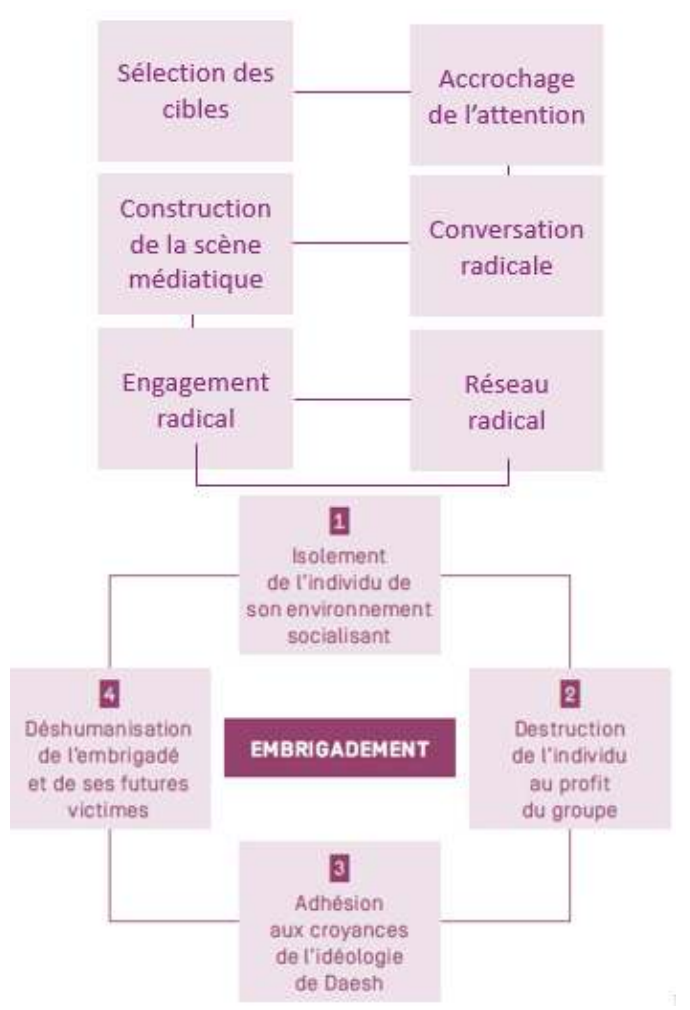

Figure2 : Schéma de l'embrigadement / engagement numérique. (Ce schéma est composé à partir du schéma de D. Bouzar CPDSI en complétant celui-ci par les caractéristiques de la conversation radicale).

Les discours de rupture et la théorie du complot agissent donc au sein de la conversation radicale comme des opérateurs de la séparation entre un « je » identifié et victimisé et un nous collectif et communautaire qui sera porté par le discours salafiste djihadiste. Les conversations radicales que nous avons identifiées respectent donc les phases 1 (isolement) et 2 (destruction de l'individu) du schéma d'embrigadement préparant ainsi le jeune à la phase reconstructive du discours 
qui s'appuyant sur une doxa structurée salafiste va montrer au jeune la voie de la rédemption qui passe par la perte de son humanité et surtout le rejet de l'humanité des autres. Cette phase présente dans la conversation numérique sera l'objet d'une autre publication.

Les réseaux sociaux et l'ensemble des dispositifs interactifs du Web 2.0 sont utilisés volontairement par les recruteurs salafistes pour organiser une scène devant conduire vers une conversion (changement de quelque chose en quelque chose d'autre). Cette démarche n'est pas de l'ordre de l'emprise mentale mais bien de la conversation structurée dans un jeu engagement / endoctrinement où le sujet est acteur et agit dans un jeu complexe de communication qui favorise la manipulation.

\section{$R \cdot E \cdot F \cdot E \cdot R \cdot E \cdot N \cdot C \cdot E \cdot S$}

AUBERT A. (2009). La société civile et ses médias. Quand le public prend la parole. Paris, Éd. Le Bord de l'eau, coll. INA.

BEAUVOIS J-L, JOULE ROBERT V. (1988). « La psychologie de la soumission ». La Recherche, $\mathrm{n}^{\circ} 202$.

BOUDON R. (1990). L'art de se persuader des idées douteuses, fragiles ou fausses. Paris, Fayard.

BOUGNOUX D. (1995). La communication contre l'information. Paris, Hachette.

BOUZAR, D. (2015). La vie après Daech. Éditions ouvrières, éditions de l'atelier BRETON P. (1996). L'argumentation dans la communication. Paris, La Découverte.

BRETON, P. (2008). Convaincre sans manipuler. Apprendre à argumenter. Paris : La Découverte. DACHEUX E. (1994). Les stratégies de communication persuasive dans l'union européenne. Paris, L'Harmattan.

DUCOL, B. (2015). « Comment le jihadisme est-il devenu numérique ? Évolutions, tendances et ripostes ». Sécurité et stratégie, vol. 20, n 1. HEINKEL, W., \& MACE, A. (2011). "Homegrown Islamist terrorism: Assessing the threat". Journal of Public and International Affairs, 22, 109-136. Retrieved from https://www.princeton.edu/jpia/past-issues-1/2011-1/JPIA2011-2. pdf\#page $=109$

LOHISSE, J. (2006). La communication : de la transmission à la relation, deuxième édition revue et augmentée par Annabelle Klein. Bruxelles, De Boeck

NESSER, P. (2006). “Jihadism in Western Europe After the Invasion of Iraq: Tracing 
Motivational Influences from the Iraq War on Jihadist Terrorism in Western Europe". Studies in Conflict \& Terrorism, 29(4), 323-342. http:// doi.org/10.1080/10576100600641899

SCHILS, N. \&J. LAFFINEUR. (2013). Comprendre et expliquer le rôle des réseaux sociaux dans la formation de l'extrémisme violent. Gent et Université Catholique de Louvain, BELSPO. http://www.belspo.be/belspo/fedra/ TA/synTA043 fr.pdf

SMITH, A. (2009). Démystifier la radicalisation. Gendarmerie royale du Canada

STAHELSKI, A. (2005). "Terrorists are made not born: Creating terrorists using social psychological conditioning". Cultic Studies Review, 4(1), 1-10. Retrieved from http://www.homelandsecurity.org/journal/articles/stahelski.html

VELDHUIS, T., \& STAUN, J. (2009). Islamist radicalisation: a root cause model. The Hague: Netherlands Institute of International Relations Clingendael. Retrieved from http:/www. dcism.dk/graphics/_IO_indsatsomraader/Religion_og_social_konflikt_og_Mellemosten/ Islamist Radicalisation.Veldhuis and Staun.pdf WATZLAWICK, P. (1980). Le langage du changement. Éd. du Seuil, Paris.

\section{$R \cdot E ́ \cdot S \cdot U \cdot M \cdot E ́$}

La radicalisation des jeunes a toujours une dimension numérique et si l'accès aux ressources terroristes est l'objet d'une stratégie calculée d'identification et de suppression dans les différents pays du monde, encore très peu d'actions sont entreprises pour réguler les réseaux sociaux dans ce domaine. Certes, les grands opérateurs que sont Google, Facebook, Twitter font un travail considérable de suppression des comptes clairement identifiés comme djihadistes, mais une grande partie des actions d'accroche et de conviction / embrigadement se réalisent dans le domaine conversationnel. Dans le cadre d'une recherche qualitative conduite entre 2014 et 2016 sur des jeunes en cours de radicalisation ou repentis, nous avons étudié la dynamique d'enrôlement numérique au cœur des réseaux sociaux. Tous les témoignages de jeunes radicalisés montrent qu'une partie du processus de recrutement passe par une stratégie de contact à partir de marqueurs sémantiques utilisés par les recruteurs pour construire une stratégie d'approche, d'accroche, de séduction numérique qui isole l'individu et qui construit une situation d'apprentissage paradoxal que nous nommons « éducation sombre » car elle engage le sujet contre sa conscience dans une situation de communication paradoxale qui va l'engager dans un abandon volontaire du jugement personnel.

\author{
Abstract \\ Study of radicalization processes within social \\ networks: role of complotist arguments and \\ rupture discourses \\ Radicalization of young people always carry
}


a numerical dimension. If the access to online terrorist resources is now being targeted by calculated strategies of identification and suppression in many countries, very little is being done to regulate Social networks in this area. Yet all testimonies of radicalized young people show that part of the recruitment process involves contact strategy based on semantic markers used by recruiters as part of a larger methodology of approach, attachment and conviction that isolates individuals. In this discursive indictrination strategy, conspiracy theories act in synergy with other themes steming from rupture discourses to enable a questioning process and the search for a hidden truth within young subjects. 